\title{
Accurate variational results for the symmetric periodic Anderson model in one, two, and three dimensions
}

\author{
Zs. Gulácsi, R. Strack, and D. Vollhardt \\ Institut für Theoretische Physik C, Technische Hochschule Aachen, D-5100 Aachen, Federal Republic of Germany
}

(Received 5 October 1992)

\begin{abstract}
The ground-state energy, hybridization energy, momentum distributions, local magnetic moments, and magnetic phase diagram of the symmetric periodic Anderson model are calculated using a correlated wave function in $d=1,2$, and 3 dimensions. The wave function is obtained by applying a Gutzwiller projector on a recently proposed single-particle product state with spin-density wave order. Evaluations are performed with an approximate scheme that becomes exact in high dimensions. Comparison with Monte Carlo data by Blankenbecler et al. for $d=1$ shows that the results are remarkably accurate for all values of the coupling. Hence the results for $d=2,3$ can be expected to be even more accurate and may serve as benchmarks for future numerical work in $d>1$.
\end{abstract}

\section{INTRODUCTION}

Heavy fermion systems are well-known to exhibit extraordinary thermodynamic, magnetic and transport properties, ${ }^{1}$ whose origin is not yet sufficiently understood. Microscopic investigations of these systems are generally based on the periodic Anderson model (PAM) Hamiltonian

$$
\begin{aligned}
\hat{H}= & \sum_{\mathbf{k} \sigma} \epsilon_{\mathbf{k}} \hat{n}_{\mathbf{k} \sigma}^{c}+E_{f} \sum_{\mathbf{k} \sigma} \hat{n}_{\mathbf{k} \sigma}^{f}+V \sum_{\mathbf{k} \sigma}\left(\hat{f}_{\mathbf{k} \sigma}^{\dagger} \hat{c}_{\mathbf{k} \sigma}+\hat{c}_{\mathbf{k} \sigma}^{\dagger} \hat{f}_{\mathbf{k} \sigma}\right) \\
& +U \sum_{\mathbf{i}} \hat{n}_{\mathbf{i} \uparrow}^{f} \hat{n}_{\mathbf{i} \downarrow}^{f},
\end{aligned}
$$

which describes a band of noninteracting conduction $(c)$ electrons with dispersion $\epsilon_{\mathrm{k}}=-2 t \sum_{i=1}^{d} \cos k_{i}$ that are hybridized via the matrix element $V$ (Ref. 2) with static $f$ electrons of energy $E_{f}$; the latter interact with each other by a Hubbard interaction. In (1) $\hat{n}_{\mathbf{k} \sigma}^{c}=\widehat{c}_{\mathbf{k} \sigma}^{\dagger} \widehat{c}_{\mathbf{k} \sigma}$, $\hat{n}_{\mathbf{k} \sigma}^{f}=\hat{f}_{\mathbf{k} \sigma}^{\dagger} \hat{f}_{\mathbf{k} \sigma}$ are density operators in momentum space, while $\hat{n}_{\mathrm{i} \sigma}^{c}=\widehat{c}_{\mathrm{i} \sigma}^{\dagger} \widehat{c}_{\mathrm{i} \sigma}, \hat{n}_{\mathrm{i} \sigma}^{f}=\hat{f}_{\mathrm{i} \sigma}^{+} \hat{f}_{\mathrm{i} \sigma}$ are local densities. In this paper we only address the symmetric PAM, where $E_{f}=-U / 2$ and the total electron density $n=2$.

The PAM defines a complicated quantum-mechanical many-body problem for which an exact solution does not even exist in $d=1$, except in a restricted parameter range. ${ }^{3}$ In such a situation the application of variational methods has proved to be very valuable. In particular, the use of variational wave functions, which may be used even in situations when standard perturbation fails or is not tractable, often leads to considerable insight. For Hubbard-type models, such as the PAM, the simplest and most well-known correlated wave function is the Gutzwiller wave function ${ }^{4}$

$$
\left|\Psi_{G}\right\rangle=g^{\hat{D}}\left|\Psi_{0}\right\rangle \text {. }
$$

It is constructed from the exact wave function of the noninteracting system, $\left|\Psi_{0}\right\rangle$, by applying the Gutzwiller projector onto $\left|\Psi_{0}\right\rangle$ to reduce the overall number of doubly occupied sites, $\langle\hat{D}\rangle$, at $U>0$. In the case of the PAM, where $\hat{D}^{f}=\hat{n}_{\mathbf{i} \uparrow}^{f} \hat{n}_{\mathrm{i} \downarrow}^{f}$, analytic evaluations ${ }^{5-7}$ are only possible within the Gutzwiller approximation, ${ }^{4,8}$ which is known to yield the correct evaluation for high dimensions, $d \rightarrow \infty .^{9,10}$ The results obtained thereby for the ground-state energy are given by ${ }^{5}$

$$
\frac{E_{\mathrm{Gutz}}}{L}=\epsilon_{0}-\frac{U}{2}-\frac{W}{2} \exp \left[-\frac{U W}{8 V^{2}}\right],
$$

with $L$ as the number of lattice sites and $\epsilon_{0}$ and $W$ as the average energy and bandwidth of the noninteracting $c$ electrons, respectively. This result, which is characterized by an exponentially small binding energy, has the form known from the single-impurity Kondo problem. ${ }^{11}$ While it gives a very good description at small $U$, it fails to reproduce the much more important large. $U$ limit, since it does not yield the (rather trivial) second-order shift $\propto-V^{2} / U$. Indeed, in the symmetric case straightforward perturbation theory in $V$ yields ${ }^{12-15}$

$$
\frac{E_{\text {exact }}}{L}=\epsilon_{0}-\frac{U}{2}-\frac{2 V^{2}}{U}, \quad U \gg\left|\epsilon_{0}\right| .
$$

The presence of a nonanalytic, Kondo-like contribution to the ground-state energy of the symmetric PAM has so far not been proved rigorously. In particular, the exact result for the asymmetric PAM in $d=1$, valid in a restricted parameter range, ${ }^{3}$ does not contain such a term.

To be able to describe the large- $U$ behavior correctly, Strack and Vollhardt ${ }^{15}$ recently proposed a nonGutzwiller-type variational wave function for the PAM with antiferromagnetically ordered $f$ electrons, which has the form

$$
\left|\Psi_{\mathrm{SV}}\right\rangle=\hat{C}|c \mathrm{FS}\rangle \otimes|f \mathrm{SDW}\rangle \text {. }
$$

It consists of a starting wave function which is a product state of a Fermi sea of $c$ electrons, $\mid c$ FS $\rangle$, and a HartreeFock spin density wave for the $f$ electrons. The correlation operator $\hat{C}=\widehat{C}_{2} \widehat{C}_{1}$ is given by

(c) 1993 The American Physical Society 


$$
\begin{aligned}
& \hat{C}_{1}=\exp \left[\sum_{\mathbf{k} \sigma} \widetilde{V}_{\mathbf{k}}\left(\hat{f}_{\mathbf{k} \sigma}^{\dagger} \widehat{c}_{\mathbf{k} \sigma}+\widehat{c}_{\mathbf{k} \sigma}^{\dagger} \hat{f}_{\mathbf{k} \sigma}\right)\right], \\
& \hat{C}_{\dot{2}}=\exp \left[\sum_{\mathbf{k} \sigma} \widetilde{\epsilon}_{\mathbf{k}} \hat{n}_{\mathbf{k} \sigma}^{f}\right],
\end{aligned}
$$

with $\widetilde{V}_{\mathrm{k}}$ and $\widetilde{\epsilon}_{\mathrm{k}}$ as $\mathbf{k}$-dependent variational functions. In contrast to (2) the interactions are more or less already incorporated in the starting wave function, while the operator $\hat{C}_{1}$ introduces the hybridization and $\hat{C}_{2}$ controls the quantum-mechanism motion of the $f$ electrons in $\mid f$ SDW $\rangle$ (note that $\ln \hat{C}_{2}$ has the form of a kinetic energy of $f$ electrons). Due to the structure of $\hat{C}$, the wave function (5) can be written as a product state ${ }^{15}$

$$
\left|\Psi_{\mathrm{sv}}\right\rangle=\prod_{\mathbf{k} \sigma} \hat{F}_{\mathbf{k} \sigma}\left[\widetilde{\epsilon}_{\mathbf{k}}, \widetilde{V}_{\mathbf{k}}, u_{\mathbf{k}}, v_{\mathbf{k}}\right]|0\rangle,
$$

where the prime indicates that the $\mathbf{k}$ values are restricted to $\epsilon_{\mathbf{k}} \leq 0$ and $\widehat{F}_{\mathbf{k} \rho}$ is a linear superposition of $\widehat{c}_{\mathbf{k} \sigma}^{\dagger} \widehat{f}_{\mathbf{k} \sigma}^{\dagger}$,

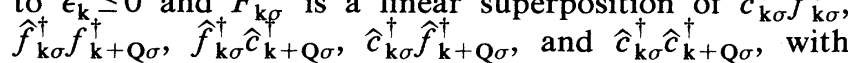
$\mathbf{Q}=(\pi, \ldots, \pi)$ as half a reciprocal lattice vector. Since (5) is a two-particle product state, all expectation values in terms of $\left|\Psi_{\mathrm{SV}}\right\rangle$ can be calculated analytically for arbitrary dimension $d$. The variational functions $u_{\mathrm{k}}, v_{\mathrm{k}}, \boldsymbol{\epsilon}_{\mathrm{k}}$ and $\widetilde{V}_{k}$ have to be determined from the minimum of the ground-state energy. It is quite easy to see that the ground-state energy of the PAM obtained with (5) has indeed the correct limiting behavior at large $U$ [which is the physically relevant regime for the PAM shown in (4)], and even at small $U .{ }^{15}$ However, for intermediate $U$ values the energy is considerably higher than found numerically. ${ }^{12}$ Subsequently, Brenig and MüllerHartmann ${ }^{16}$ noted that the wave function (5) can be improved by adding the term $\widehat{c}_{\mathbf{k}+\mathbf{Q} \sigma}^{\dagger} \widehat{f}_{\mathbf{k}+\mathbf{Q} \sigma}^{\dagger}$ to $\widehat{F}_{\mathbf{k} \sigma}$ in (7), whereby the $\mathbf{k}$ dependence of $c$ and $f$ electrons is made symmetric. In this way one obtains the following wave function, which is the most general two-particle product state permitting antiferromagnetic order

$$
\begin{aligned}
\left|\Psi_{\mathrm{BMH}}\right\rangle=\prod_{\mathbf{k} \sigma}{ }^{\prime} & {\left[\widetilde{u}_{\mathbf{k}} \widehat{c}_{\mathbf{k} \sigma}^{\dagger} \hat{f}_{\mathbf{k} \sigma}^{\dagger}+\widetilde{z}_{\mathbf{k}} \hat{c}_{\mathbf{k}+\mathbf{Q} \sigma}^{\dagger} \hat{f}_{\mathbf{k}+\mathbf{Q} \sigma}^{\dagger}\right.} \\
& +\sigma\left(\widetilde{v}_{\mathbf{k}} \hat{c}_{\mathbf{k}+\mathbf{Q} \sigma}^{\dagger} \hat{f}_{\mathbf{k} \sigma}^{\dagger}+\widetilde{w}_{\mathbf{k}} \widehat{c}_{\mathbf{k} \sigma}^{\dagger} \hat{f}_{\mathbf{k}+\mathbf{Q} \sigma}^{\dagger}\right. \\
& \left.\left.+\widetilde{x}_{\mathbf{k}} \widehat{c}_{\mathbf{k}+\mathbf{Q} \sigma}^{\dagger} \widehat{c}_{\mathbf{k} \sigma}^{\dagger}+\widetilde{y}_{\mathbf{k}} \hat{f}_{\mathbf{k}+\mathbf{Q} \sigma}^{\dagger} \hat{f}_{\mathbf{k} \sigma}^{\dagger}\right)\right]|0\rangle,
\end{aligned}
$$

where $\widetilde{u}_{\mathrm{k}}, \widetilde{v}_{\mathrm{k}}, \widetilde{w}_{\mathrm{k}}, \widetilde{x}_{\mathrm{k}}, \widetilde{y}_{\mathrm{k}}$, and $\widetilde{z}_{\mathrm{k}}$ are variational functions which have to make the ground-state energy minimal. The authors also pointed out ${ }^{16}$ that, under the constraint

$$
-\widetilde{u}_{\mathbf{k}} \widetilde{z}_{\mathbf{k}}+\widetilde{v}_{\mathbf{k}} \widetilde{w}_{\mathbf{k}}+\widetilde{x}_{\mathbf{k}} \widetilde{y}_{\mathbf{k}}=0
$$

Eq. (8) is equivalent to a single Slater determinant spindensity wave (SDW) ground-state wave function

$$
\begin{aligned}
\left|\Psi_{\mathrm{SDW}}\right\rangle=\prod_{\mathbf{k} \sigma} \prod_{n=1}^{\prime}\left[\widetilde{\alpha}_{\mathbf{k} n} \widehat{c}_{\mathbf{k} \sigma}^{\dagger}+\widetilde{\beta}_{\mathbf{k} n} \widehat{f}_{\mathbf{k} \sigma}^{\dagger}\right. \\
\left.+\sigma\left(\widetilde{\gamma}_{\mathbf{k} n} \widehat{c}_{\mathbf{k}+\mathbf{Q} \sigma}^{\dagger}+\widetilde{\delta}_{\mathbf{k} n} \widehat{f}_{\mathbf{k}+\mathbf{Q} \sigma}^{\dagger}\right)\right]|0\rangle,
\end{aligned}
$$

where $\widetilde{\alpha}_{\mathbf{k} n}, \widetilde{\beta}_{\mathbf{k} n}, \widetilde{\gamma}_{\mathbf{k} n}$, and $\widetilde{\delta}_{\mathbf{k} n}$ are variational functions and $n=1,2$ labels two orthogonal combinations. It turns out that, in the case of the symmetric PAM considered here, the optimal values of the variational functions automatically fulfill the constraint (9). Hence, for the symmetric PAM the wave functions (8) and (10) are equivalent. The expectation value of (1) in terms of (8) and (10) can also be expressed in closed form and the minimization can be performed for any dimension. ${ }^{16}$ At intermediate $U$ values the energy is found to be considerably lower than that obtained with (5). Its good overall agreement with the quantum Monte-Carlo calculations by Blankenbecler et al..$^{12}$ is quite remarkable in view of the fact that $(10)$ is only a single-particle product wave function, i.e., does not contain true two-particle correlation effects at all.

At this point it is clear how to proceed to obtain an even better variational wave function for the PAM: We have to consider the wave function (10) as a starting wave function itself. By applying the Gutzwiller correlator we obtain a wave function

$$
|\Psi\rangle=g^{\hat{D}^{f}}\left|\Psi_{\text {SDW }}\right\rangle,
$$

with $g$ as an additional variational parameter, where two-particle correlation effects are now explicitly included. Analytic evaluations of expectation values in terms of (11) can now be performed in closed form only in the limit $d=\infty \cdot{ }^{17,18}$ However, from our earlier experience ${ }^{18-20}$ we may expect that, by evaluating the analytic $d=\infty$ result with the $d$-dimensional density of states, accurate results can even be obtained down to $d=1$, which is obviously the most unfavorable limit for the $d=\infty$ approach.

The aim of our paper is therefore to evaluate a number of ground-state quantities, e.g., the ground-state and hybridization energy, as well as the momentum distributions and the local moments of the $c$ and $f$ electrons of the symmetric PAM in terms of the improved correlated spin-density wave function in $d=12$, and 3 , using the large- $d$ approach.

\section{EXPECTATION VALUES IN TERMS OF THE CORRELATED SPIN-DENSITY WAVE FUNCTION IN $\boldsymbol{d}=\infty$}

Expectation values of operators in terms of Gutzwiller-type wave functions (2), may be calculated within a diagrammatic theory. ${ }^{21}$ In the limit $d \rightarrow \infty$ the ensuing collapse of diagrams greatly simplifies the evaluation, but it remains nontrivial because Hartree bubbles survive. Gebhard ${ }^{19}$ developed a particularly efficient diagrammatic formalism which, for Gutzwiller-type wave functions (2) in $d=\infty$, allows one to obtain expectation values exactly and in closed form. This is achieved by writing the starting wave function $\left|\Psi_{0}\right\rangle$ in (2) in the form

$$
\left|\Psi_{0}\right\rangle=g^{-\sum_{\mathbf{i} \sigma} \mu_{\mathrm{i} \sigma} \hat{n}_{\mathrm{i} \sigma}}\left|\Phi_{0}\right\rangle,
$$

where $\left|\Phi_{0}\right\rangle$ is again an arbitrary one-particle wave function and the $\mu_{\mathrm{i} \sigma}$ are explicit functions of $g$ and the local density

$$
n_{\mathrm{i} \sigma}=\left\langle\Phi_{0}\left|\hat{n}_{\mathrm{i} \sigma}\right| \Phi_{0}\right\rangle /\left\langle\Phi_{0} \mid \Phi_{0}\right\rangle .
$$

The projection operator in (12) corresponds to a gauge transformation by which the local chemical potentials 
can be chosen such that all Hartree bubbles disappear in $d=\infty$. Consequently, in $d=\infty$ diagrams vanish completely and results are obtained without the calculation of a single graph. ${ }^{19}$ In particular, all expectation values can be obtained exactly in $d=\infty:^{19,22}$

$\left\langle\hat{f}_{\mathrm{i} \sigma}^{\dagger} \hat{f}_{\mathbf{j} \sigma}\right\rangle=\sqrt{q_{\mathrm{i} \sigma}} \sqrt{q_{\mathrm{j} \sigma}}\left\langle\hat{f}_{\mathrm{i} \sigma}^{\dagger} \hat{f}_{\mathrm{j} \sigma}\right\rangle_{0}+\delta_{\mathrm{ij}}\left(1-q_{\mathrm{j} \sigma}\right) n{ }_{\mathbf{j} \sigma}^{f, 0}$,

$\left\langle\hat{c}_{\mathbf{i} \sigma}^{\dagger} \hat{f}_{\mathbf{j} \sigma}\right\rangle=\sqrt{q_{\mathbf{j} \sigma}}\left\langle\hat{c}_{\mathbf{i} \sigma}^{\dagger} \hat{f}_{\mathbf{j} \sigma}\right\rangle_{0}$,

$\left\langle\widehat{c}_{\mathbf{i} \sigma}^{\dagger} \widehat{c}_{\mathbf{j} \sigma}\right\rangle=\left\langle\hat{c}_{\mathbf{i} \sigma}^{\dagger} \hat{c}_{\mathbf{j} \sigma}\right\rangle_{0}$,

where $\langle\cdots\rangle=\langle\Psi|\cdots| \Psi\rangle /\langle\Psi \mid \Psi\rangle$ and

$$
\langle\cdots\rangle_{0}=\left\langle\Phi_{0}|\cdots| \Phi_{0}\right\rangle /\left\langle\Phi_{0} \mid \Phi_{0}\right\rangle .
$$

The $q$ factors are given by

$$
\begin{aligned}
q_{\mathrm{i} \sigma}=\frac{1}{\left[n_{\mathrm{i} \sigma}^{f, 0}\left(1-n_{\mathrm{i} \sigma}^{f, 0}\right)\right]} & {\left[\sqrt{\left(1-n_{\mathrm{i}}^{f, 0}+d_{\mathrm{i}}^{f}\right)\left(n_{\mathrm{i} \sigma}^{f, 0}-d_{\mathrm{i}}^{f}\right)}\right.} \\
& \left.+\sqrt{\left.d_{\mathrm{i}}^{f\left(n_{\mathrm{i}}^{f, 0}-\sigma\right.}-d_{\mathrm{i}}^{f}\right)}\right]^{2}
\end{aligned}
$$

where $n_{\mathrm{j} \sigma}^{f, 0}=\left\langle\hat{n}_{\mathbf{j} \sigma}^{f}\right\rangle_{0}, n_{\mathrm{j}}^{f, 0}=n_{\mathrm{j} \uparrow}^{f, 0}+n_{\mathrm{j} \downarrow}^{f, 0}$ and $d d_{\mathrm{j}}^{f}=\left\langle\hat{D}_{\mathbf{j}}^{f}\right\rangle$. The variational parameter $g$ is connected with the double occupancy $d_{\mathrm{j}}^{f}$ by

$$
g^{2}=\frac{d_{\mathrm{j}}^{f}\left(1-n_{\mathrm{j}}^{f, 0}+d_{\mathrm{j}}^{f}\right)}{\left[n_{\mathrm{j} \uparrow}^{f, 0}-d_{\mathrm{j}}^{f}\right]\left[n_{\mathrm{j} \downarrow}^{f, 0}-d_{\mathrm{j}}^{f}\right]} .
$$

In order to be able to apply the above results to the Gutzwiller-correlated SDW (11), we first have to determine the new single-particle wave function $\left|\Phi_{0}\right\rangle$ in (12). For computational reasons we prefer to calculate with $\left|\Psi_{0}\right\rangle \equiv\left|\Psi_{\mathrm{BMH}}\right\rangle$ [plus the constraint (9)], rather ${ }^{16}$ than $\left|\Psi_{\text {SDW }}\right\rangle$ in (11). Hence we have to determine

$$
\left|\Phi_{0}\right\rangle=g^{\sum_{\mathrm{i} \sigma} \mu_{\mathrm{i} \sigma} \hat{n}_{\mathrm{i} \sigma}^{f}}\left|\Psi_{\mathrm{BMH}}\right\rangle .
$$

It is easy to show that $\left|\Phi_{0}\right\rangle$ has exactly the same form as $\left|\Psi_{\mathrm{BMH}}\right\rangle$, albeit with new variational functions $u_{\mathrm{k}}, v_{\mathrm{k}}, w_{\mathrm{k}}$, $x_{\mathrm{k}}, y_{\mathrm{k}}, z_{\mathrm{k}}$ (the latter are functions of the old parameters $\widetilde{u}_{k}$, etc., as well as of $g$ and $\mu_{i \sigma}$ ). Also the constraint (9)

$$
-u_{\mathrm{k}} z_{\mathrm{k}}+v_{\mathrm{k}} w_{\mathrm{k}}+x_{\mathrm{k}} y_{\mathrm{k}}=0
$$

is fulfilled. We take the parameters $u_{\mathrm{k}}$, etc., as new variational functions.

The expectation value of the Hamiltonian with respect to the Gutzwiller-correlated spin-density wave is then given by

$$
\begin{aligned}
\langle\hat{H}\rangle= & \sum_{\mathbf{k} \sigma} \epsilon_{\mathbf{k}}\left\langle\hat{n}_{\mathbf{k} \sigma}^{c}\right\rangle_{0} \\
& +2 V \sqrt{q} \sum_{\mathbf{k} \sigma}\left\langle\hat{f}_{\mathbf{k} \sigma}^{\dagger} \hat{c}_{\mathbf{k} \sigma}\right\rangle_{0}-\frac{U}{2} L+U d^{f} L,
\end{aligned}
$$

where we made use of the fact that in spite of the SDW order, $q_{\mathrm{i} \sigma}$ is independent of $\mathrm{i}, \sigma$ and $d_{\mathrm{i}}^{f}$ is independent of i. The uncorrelated quantities $\langle\cdots\rangle_{0}$ were already calculated by Brenig and Müller-Hartmann, ${ }^{16}$ whose notation we will use. The renormalization factor $\sqrt{q}$ has the form $\sqrt{q}=\frac{\sqrt{d^{f}}}{\sqrt{\frac{1}{4}-4 \Delta_{f}^{2}}}\left[\left(\frac{1}{2}-d^{f}+2 \Delta_{f}\right)^{1 / 2}+\left(\frac{1}{2}-d^{f}-2 \Delta_{f}\right)^{1 / 2}\right]$,

where

$\Delta_{f}=\frac{1}{L} \sum_{\mathbf{k}}^{\prime}\left\langle\hat{f}_{\mathbf{k} \sigma}^{\dagger} \hat{f}_{\mathbf{k}+\mathbf{Q} \sigma}\right\rangle_{0}=\frac{1}{L} \sum_{\mathbf{k}}^{\prime} \frac{u_{\mathbf{k}} w_{\mathbf{k}}+v_{\mathbf{k}} z_{\mathbf{k}}}{N_{\mathbf{k}}}$,

with

$$
N_{\mathrm{k}}=v_{\mathrm{k}}^{2}+w_{\mathrm{k}}^{2}+u_{\mathrm{k}}^{2}+z_{\mathrm{k}}^{2}+x_{\mathrm{k}}^{2}+y_{\mathrm{k}}^{2} \equiv 1 .
$$

Here the prime on the $\mathbf{k}$ sum implies the restriction $\epsilon_{\mathrm{k}} \leq 0$. The parameter $\Delta_{f}$ determines the amplitude of the SDW

$$
\left\langle\hat{n}_{\mathbf{j} \sigma}^{f}\right\rangle_{0}=\frac{1}{2}+2 \Delta_{f} \sigma \cos (\mathbf{Q} \cdot \mathbf{j})
$$

Hence $\Delta_{f}=0$ refers to the nonmagnetic phase. Since the wave function (10) is symmetric in the $c$ and $f$ electrons, a SDW formation of the $f$ electrons implies that the conduction electrons form a SDW, too. In analogy to (19b) and (19c) one finds

$$
\left\langle\hat{n}_{\mathbf{j} \sigma}^{c}\right\rangle_{0}=\frac{1}{2}+2 \Delta_{c} \sigma \cos (\mathbf{Q} \cdot \mathbf{j}),
$$

where

$\Delta_{c}=\frac{1}{L} \sum_{\mathbf{k}}^{\prime}\left\langle\widehat{c}_{\mathbf{k} \sigma}^{\dagger} \widehat{c}_{\mathbf{k}+\mathbf{Q} \sigma}\right\rangle_{0}=\frac{1}{L} \sum_{\mathbf{k}}^{\prime} \frac{u_{\mathbf{k}} v_{\mathbf{k}}+w_{\mathbf{k}} z_{\mathbf{k}}}{N_{\mathbf{k}}}$.

The local magnetic moments $\left\langle\hat{m}_{\mathrm{j}, z}\right\rangle=\left\langle\hat{n}_{\mathrm{j} \uparrow}-\hat{n}_{\mathrm{j} \downarrow}\right\rangle$ of the $c$ and $f$ electrons are therefore given by

$$
\begin{aligned}
& \left\langle\hat{m}_{\mathbf{j}, z}^{c}\right\rangle=4 \Delta_{c} \cos (\mathbf{Q} \cdot \mathbf{j}), \\
& \left\langle\hat{m}_{\mathbf{j}, z}^{f}\right\rangle=4 \Delta_{f} \cos (\mathbf{Q} \cdot \mathbf{j}),
\end{aligned}
$$

with a total local magnetic moment of

$\left\langle\hat{m}_{\mathbf{j}, z}^{\text {tot }}\right\rangle=\left\langle\hat{m}_{\mathbf{j}, z}^{c}+\hat{m}_{\mathbf{j}, z}^{f}\right\rangle=4\left(\Delta_{c}+\Delta_{f}\right) \cos (\mathbf{Q} \cdot \mathbf{j})$.

Furthermore, the momentum distributions of the $c$ and $f$ electrons are obtained from (13a) and (13c)

$$
\begin{aligned}
& \left\langle\hat{n}_{\mathbf{k} \sigma}^{c}\right\rangle=\left\langle\hat{n}_{\mathbf{k} \sigma}^{c}\right\rangle_{0}, \\
& \left\langle\hat{n}_{\mathbf{k} \sigma}^{f}\right\rangle=\frac{1}{2}(1-q)+q\left(\hat{n}_{\mathbf{k} \sigma}^{f}\right\rangle_{0} .
\end{aligned}
$$

In terms of the new variational parameters the energy $E=\langle\hat{H}\rangle$ is given by

$$
\begin{aligned}
E= & 2 \sum_{\mathbf{k}}^{\prime} \epsilon_{\mathbf{k}} \frac{u_{\mathbf{k}}^{2}+w_{\mathbf{k}}^{2}-v_{\mathrm{k}}^{2}-z_{\mathbf{k}}^{2}}{N_{\mathrm{k}}} \\
& +8 V \sqrt{q} \sum_{\mathbf{k}}^{\prime} \frac{x_{\mathbf{k}}\left(v_{\mathbf{k}}-w_{\mathbf{k}}\right)}{N_{\mathbf{k}}}-\frac{U}{2} L+U d^{f} L .
\end{aligned}
$$

Note that in the symmetric case $x_{k}=y_{k}$. In (22) we use $d^{f}$ instead of $g$ as a new variational parameter. In order to enforce the constraints

$$
\begin{aligned}
& Q_{\mathbf{k}}^{(1)} \equiv 1-N_{\mathbf{k}}=0, \\
& Q_{\mathbf{k}}^{(2)} \equiv-u_{\mathbf{k}} z_{\mathbf{k}}+v_{\mathbf{k}} w_{\mathbf{k}}+x_{\mathbf{k}}^{2}=0,
\end{aligned}
$$


we introduce two Lagrange multipliers denoted by $\Lambda_{\mathrm{k}}^{(1)}=2 \lambda_{\mathrm{k}}$ and $\Lambda_{\mathrm{k}}^{(2)}=4 \widetilde{\lambda}_{\mathrm{k}}$ and replace $(22)$ by

$$
\widetilde{E}=E+\sum_{\mathbf{k}}^{\prime} \sum_{i=1}^{2} \Lambda_{\mathrm{k}}^{(i)} Q_{\mathrm{k}}^{(i)}
$$

This expression has to be minimized with respect to $v_{\mathrm{k}}$, $w_{\mathbf{k}}, u_{\mathbf{k}}, x_{\mathbf{k}}, z_{\mathbf{k}}$, and $d^{f}$ (see the Appendix).

The minimum of the energy is found as

$$
E=2 \sum_{\mathbf{k}}^{\prime} \lambda_{\mathbf{k}}+8 \bar{\Delta} \Delta_{f} L-\frac{U}{2} L+U d^{f} L
$$

where

$$
\begin{aligned}
& \bar{\Delta}=-\frac{\partial \ln q}{\partial \Delta} \frac{1}{2 L} \sum_{\mathbf{k}}^{\prime} x_{\mathbf{k}}^{2}, \\
& \Delta_{f}=\frac{1}{L} \sum_{\mathbf{k}}^{\prime} \frac{2 \bar{\Delta} \bar{V}^{2}\left(\epsilon_{\mathbf{k}}-\lambda_{\mathbf{k}}\right)+A_{\mathbf{k}} B_{\mathbf{k}}}{C_{\mathbf{k}}^{2}} x_{\mathbf{k}}^{2},
\end{aligned}
$$

and the parameters $A_{\mathrm{k}}, B_{\mathrm{k}}, C_{\mathrm{k}}$, and $x_{\mathrm{k}}$ are given by

$x_{\mathrm{k}}^{2}=\frac{C_{\mathrm{k}}^{2}}{2 C_{\mathrm{k}}^{2}+4 \bar{\Delta}^{2} \bar{V}^{2}+\bar{V}^{2}\left(\epsilon_{\mathrm{k}}-\lambda_{\mathrm{k}}\right)^{2}+A_{\mathrm{k}}^{2}+B_{\mathrm{k}}^{2}}$,

$A_{\mathbf{k}}=\epsilon_{\mathrm{k}} \bar{V}+\left(\epsilon_{\mathrm{k}}^{2}+p^{2}-\lambda_{\mathrm{k}} \epsilon_{\mathrm{k}}\right) \frac{\lambda_{\mathrm{k}}}{\bar{V}}$,

$B_{\mathbf{k}}=-2 \bar{\Delta} \bar{V}+\frac{\lambda_{\mathbf{k}}^{2}\left(\bar{V}^{2}-p^{2}\right)+\epsilon_{\mathbf{k}} \lambda_{\mathbf{k}}\left(4 \bar{\Delta}^{2}+p^{2}-\bar{V}^{2}\right)}{2 \bar{\Delta} \bar{V}}$

$C_{\mathbf{k}}=\bar{V}^{2}+\epsilon_{\mathrm{k}}^{2}+p^{2}-\epsilon_{\mathbf{k}} \lambda_{\mathrm{k}}$

with

$p^{2}=\sqrt{\bar{V}^{4}+4 \bar{\Delta}^{2} \epsilon_{\mathrm{k}}^{2}}$.

Furthermore, the hybridization energy

$$
E_{\mathrm{Hyb}}=\left\langle V \sum_{\mathbf{k} \sigma}\left(\hat{f}_{\mathbf{k} \sigma}^{\dagger} \hat{c}_{\mathbf{k} \sigma}+\hat{c}_{\mathbf{k} \sigma}^{\dagger} \hat{f}_{\mathbf{k} \sigma}\right)\right\rangle
$$

is obtained from

$$
E_{\mathrm{Hyb}}=-\frac{16 \bar{\Delta} L}{\partial \ln q / \partial \Delta_{f}}
$$

and the square of the local $f$-electron magnetization $\hat{m}_{\mathbf{j}, z}^{2}=\left(\hat{n}_{\mathrm{j} \uparrow}^{f}-\hat{n}_{\mathrm{j} \downarrow}^{f}\right)^{2}$, which is independent of $\mathbf{j}$, is determined by the double occupancy as

$$
\left\langle\hat{m}_{z}^{2}\right\rangle=1-2 d^{f} \text {. }
$$

We note that, due to $L\left(d^{f}-\frac{1}{2}\right)=d E / d U,\left\langle\hat{m}_{z}^{2}\right\rangle$ is a much more sensitive measure of correlation effects than the ground-state energy $E$ itself. For small $U$, where ${ }^{23}$ $E=E_{0}+C_{\mathrm{HF}} U-C_{\mathrm{Corr}} U^{2}$, with $C_{\mathrm{HF}}, C_{\mathrm{Corr}}>0$ one finds $\left\langle\hat{m}_{z}^{2}\right\rangle=\frac{1}{2}+4 C_{\text {Corr }} U$. Hence the slope of $\left\langle\hat{m}_{z}^{2}\right\rangle$ is a direct indicator of the degree to which correlation effects are included in the calculation.

For the paramagnetic case with $\Delta_{f}=\Delta_{c}=\bar{\Delta}=0$, which corresponds to the usual Gutzwiller wave function, the energy is given by

$$
E=-2 \sum_{\mathbf{k}}^{\prime} \sqrt{\epsilon_{\mathbf{k}}^{2}+32 V^{2} d^{f}\left(1-2 d^{f}\right)}-\frac{U}{2} L+U d^{f} L
$$

where $d^{f}$ is determined by the implicit equation

$$
U=32 V^{2}\left(1-4 d^{f}\right) \frac{1}{L} \sum_{\mathbf{k}}^{\prime} \sqrt{\epsilon_{\mathbf{k}}^{2}+32 V^{2} d^{f}\left(1-2 d^{f}\right)} .
$$

Moreover the hybridization energy becomes

$$
E_{\mathrm{Hyb}}=-64 V^{2} d^{f}\left(1-2 d^{f}\right) \frac{1}{L} \sum_{\mathbf{k}}^{\prime} \sqrt{\epsilon_{\mathrm{k}}^{2}+32 V^{2} d^{f}\left(1-2 d^{f}\right)} .
$$

\section{RESULTS AND DISCUSSIONS}

The expression for the ground-state energy $E$ (25), the hybridization energy $E_{\mathrm{Hyb}}(28)$, and the square of the local magnetization $\left\langle\hat{m}_{z}^{2}\right\rangle$ of the $f$ electrons (29), derived in the limit $d=\infty$, will now be evaluated in dimensions $d=12$, and 3 by using the $d$-dimensional density of states. ${ }^{18-20}$ Technically this implies an inclusion of $1 / d$ corrections to infinite order. The results will be compared with perturbation theory, Monte Carlo data, and results obtained with other variational wave functions. We will also present results for the momentum distributions $\left\langle\hat{n}_{\mathbf{k} \sigma}^{c, f}\right\rangle(21)$, and the local magnetic moments $\left\langle\hat{m}_{\mathbf{j}, z}^{c, f}\right\rangle(20 \mathrm{a})$ and $(20 \mathrm{~b})$, of the $c$ and $f$ electrons in $d=1$.

In Fig. 1 the $U$ dependence of $E, E_{\mathrm{Hyb}}$, and $\left\langle\hat{m}_{z}^{2}\right\rangle$ obtained for $d=1$ is shown for four different values of the hybridization strength $V$. To arrive at a more sensitive plot of the $U$ dependence of $E$ the energy is measured relative to the $f$-level position $-U / 2$, in units of its absolute value at $U=0,\left|E_{0}\right|$. The hybridization energy is also plotted in units of its $U=0$ value, $E_{0, \text { Hyb }}$. Both $E$ and $E_{\mathrm{Hyb}}$ tend to increase with $V$, while $\left\langle\hat{m}_{z}^{2}\right\rangle$ decreases. This holds in every dimension $d$ (see below).

In Fig. 2 the results for $d=1$ and $V / 2 t=0.5$ are compared with perturbation theory at small and large $U .^{12}$ We know already that for any dimension $d$ even the uncorrelated SDW wave function describes the small- and large- $U$ regime of the ground-state energy correctly - at least asymptotically. However, it is not a priori known what the actual size of this asymptotic regime is, or how the size increases once correlation effects are included. A qualitative answer is provided by Fig. 2(a), at least for the regime of small to intermediate $U$ values: The correlations introduced by the Gutzwiller correlator lead to a considerable lowering of $E$, in agreement with perturbation theory for small $U$. This is even more clearly seen from the slope of $\left\langle\hat{m}_{z}^{2}\right\rangle$ in Fig. 2(c): For the SDW this quantity, which measures the derivative of $E$ with respect to $U$, is constant below $U / 2 t \simeq 0.8$. This reflects the fact that $\left|\Psi_{\text {SDW }}\right\rangle$ is an uncorrelated wave function with $C_{\text {Corr }}=0$. In this case the square of the local magnetic moment becomes $U$ dependent only for $U / 2 t \gtrsim 0.8$, when a (spurious) transition to a state with antiferromagnetically ordered $f$ spins (and $c$ electrons) takes place. By contrast, the correlated SDW (11), obviously describes al- 
(a)

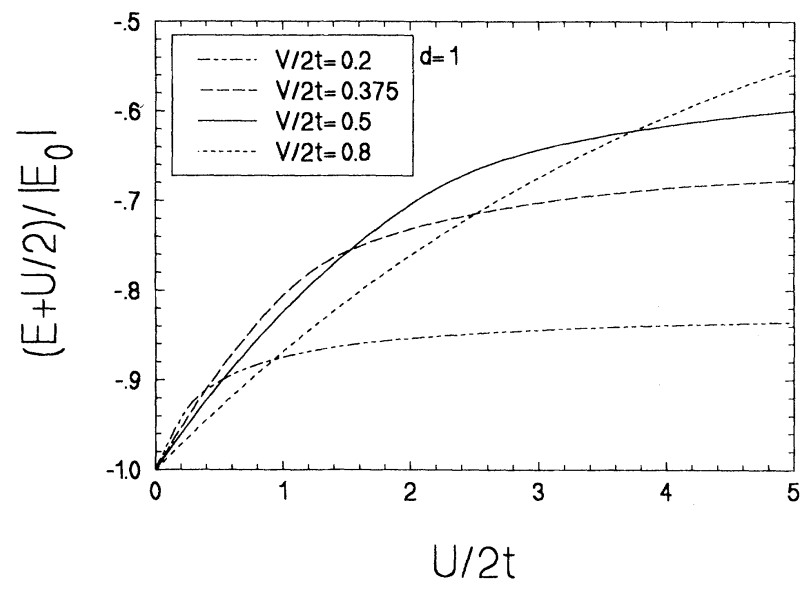

(b)

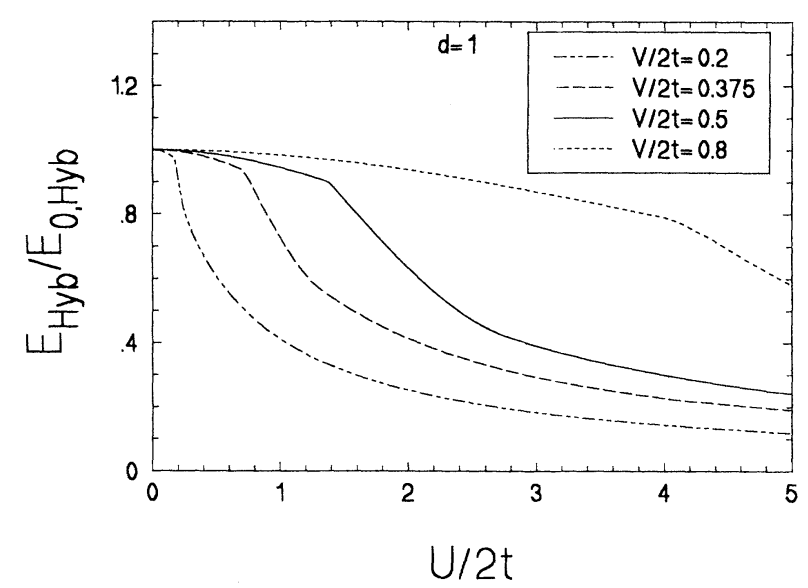

(c)

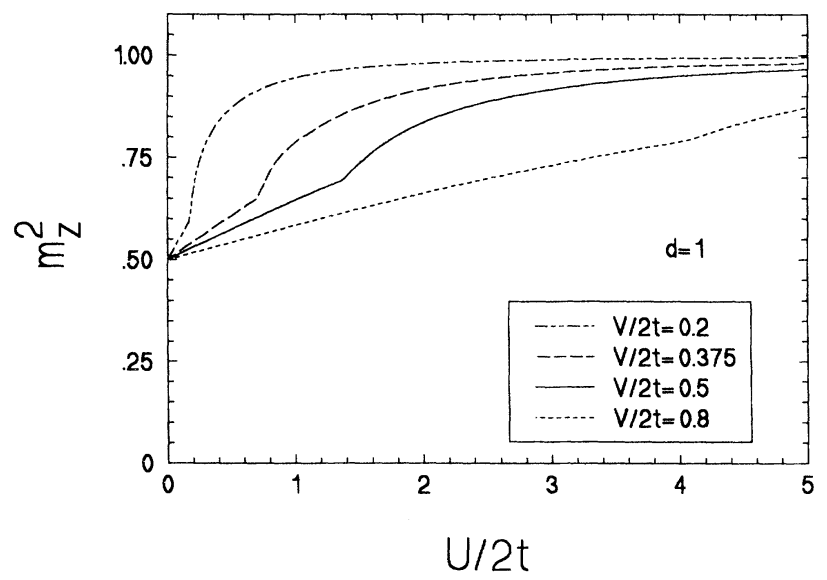

FIG. 1. (a) Ground-state energy $E$, (b) hybridization energy $E_{\mathrm{Hyb}}$, (c) square of the local $f$-electron magnetization $\left\langle\hat{m}_{z}^{2}\right\rangle$ vs $U / 2 t$ in $d=1$ for various values of the hybridization $V$ as obtained with the Gutzwiller-correlated SDW (11). (a)

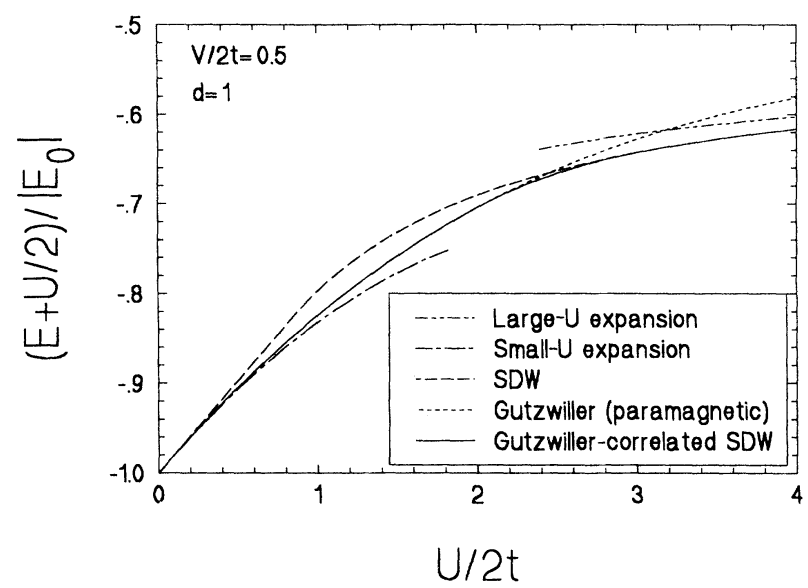

(b)

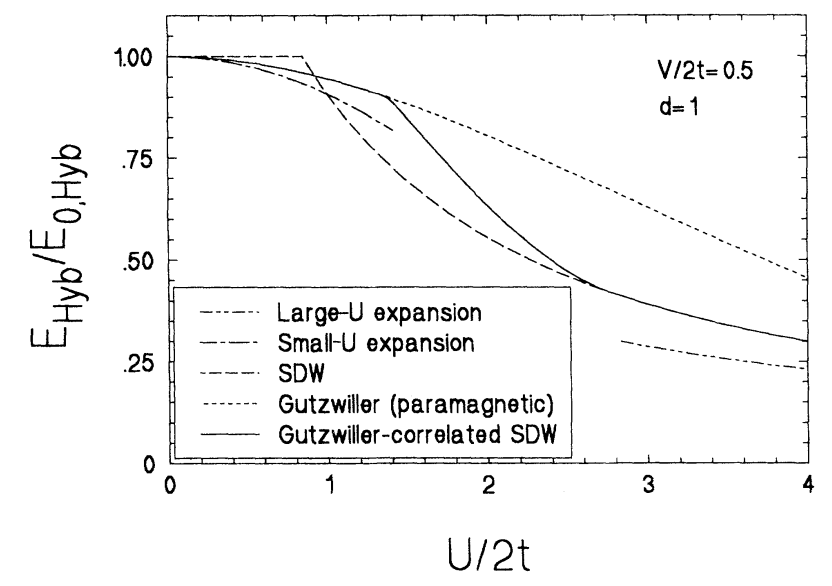

(C)

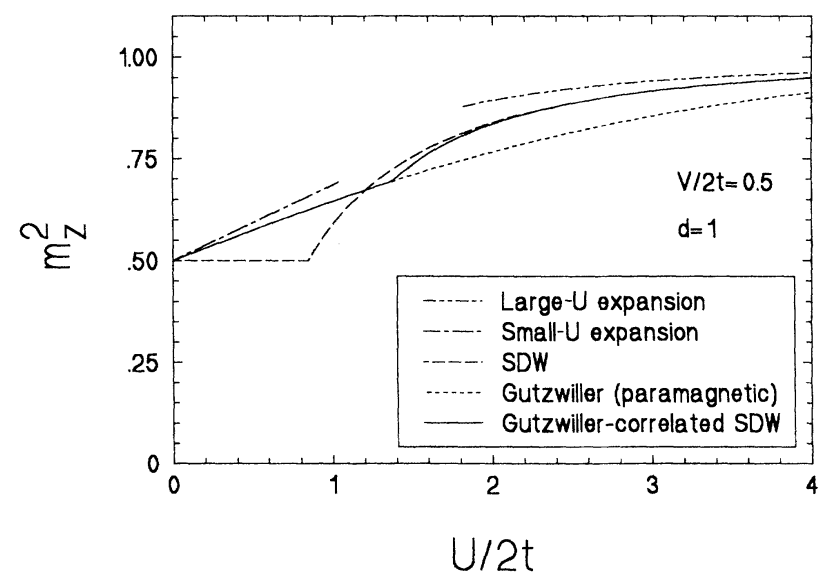

FIG. 2. The results for (a) $E$, (b) $E_{\mathrm{Hyb}}$, (c) $\left\langle\hat{m}_{z}^{2}\right\rangle$ vs $U / 2 t$ in $d=1$ for $V / 2 t=0.5$ as obtained with the Gutzwiller-correlated SDW are compared with perturbation theory at small and large $U$, as well as with the results for the uncorrelated SDW and the paramagnetic Gutzwiller wave function. 
(a)

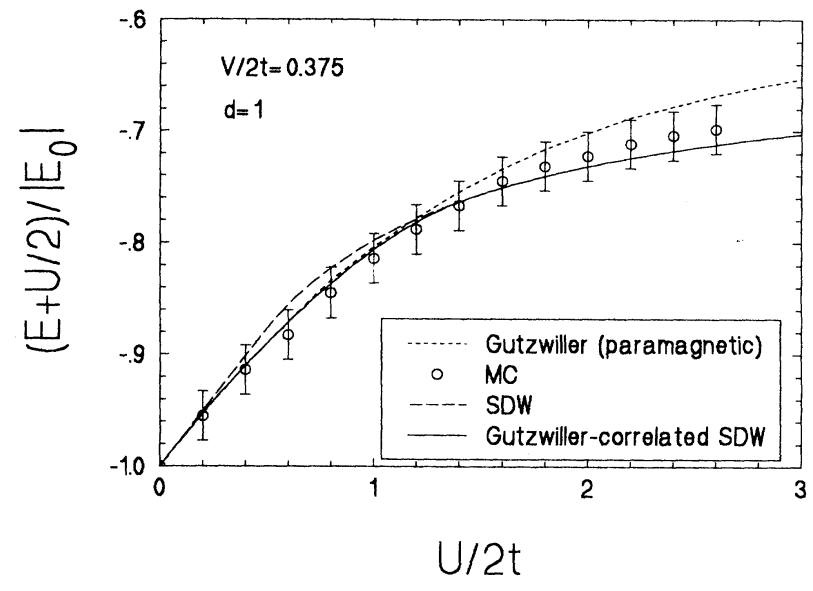

(b)

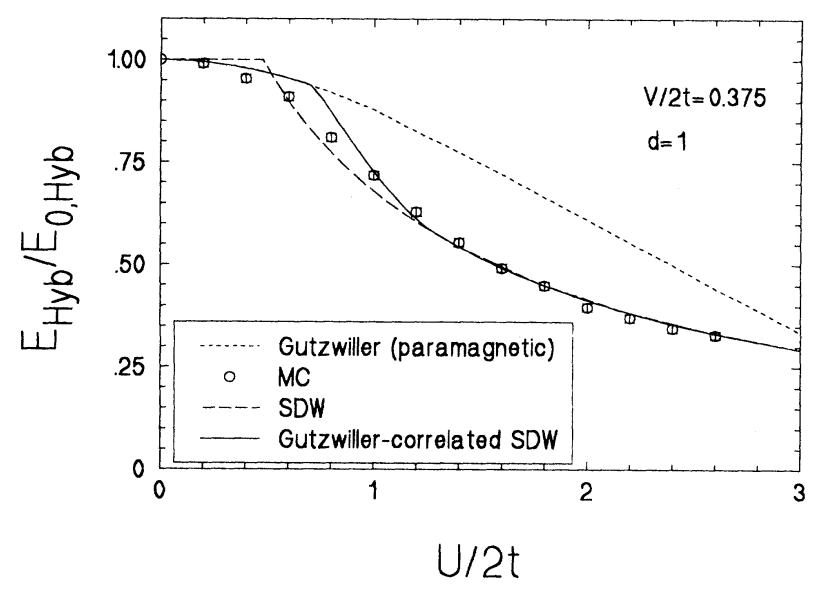

(c)

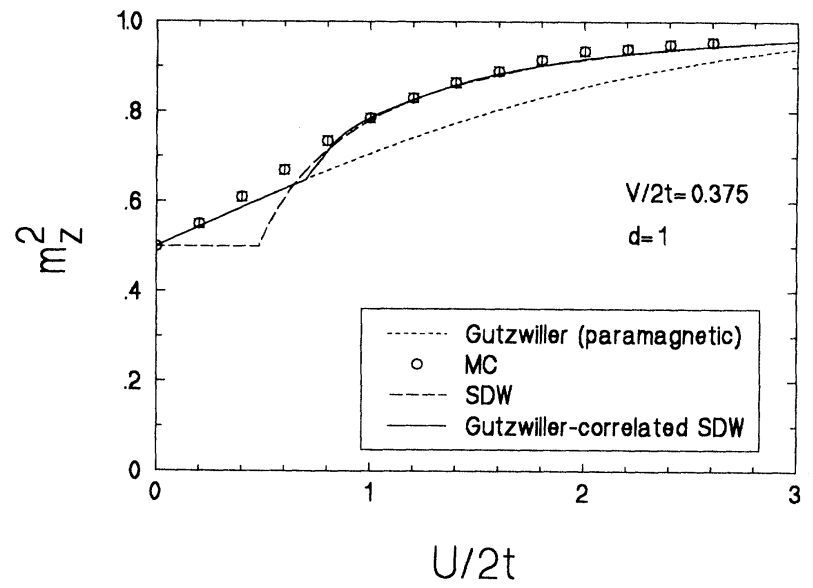

FIG. 3. The results for (a) $E$, (b) $E_{\mathrm{Hyb}}$, (c) $\left\langle\hat{m}_{z}^{2}\right\rangle$ vs $U / 2 t$ in $d=1$ for $V / 2 t=0.375$ as obtained with the Gutzwillercorrelated SDW are compared with quantum Monte Carlo data by Blankenbecler et al. (Ref. 12), as well as with the results for the uncorrelated SDW and the paramagnetic Gutzwiller wave function. The error bars correspond to the width of the data points of Ref. 12 . most the entire correlation contributions for $U / 2 t \lesssim 0.6$. For $U / 2 t \gtrsim 2$ the correlation effects due to the Gutzwiller correlator become ineffective since the antiferromagnetic correlations of the $f$ electrons (appearance of long-range order at $U / 2 t \simeq 1.4$ ) suppress their double occupancy via the opening of a gap. Of course the appearance of longrange antiferromagnetic order in $d=1$ is an artifact of the variational approach. Nevertheless it is a clear expression of the existence of strong short-range antiferromagnetic correlations between the $f$ electrons, whichon the level of the rather simple variational wave function (11) - is very well described by an actual long-range order. A similar behavior is observed for $E_{\mathrm{Hyb}}$ in Fig. 2(b). The results in Fig. 2 also clearly show that the paramagnetic Gutzwiller wave function does not provide an adequate description of the physics above $U / 2 t \simeq 1$.

For large $U$ the result for the ground-state energy obtained from perturbation theory ${ }^{12}$ lies above the variational result. Hence it is still not clear how close the energy obtained from the (correlated) SDW is to the exact result. For $d=1$ this question is answered in Fig. 3, where a comparison with the Monte-Carlo data of Blankenbecler et al. ${ }^{12}$ for $V / 2 t=0.375$ is shown. Clearly the correlated SDW describes the numerical results very well for all $U$ values. For $U / 2 t \lesssim 1$ the correlation effects are seen to be very important for all quantities, while for $U / 2 t \gtrsim 1$ excellent agreement is reached already with the uncorrelated SDW. This shows even more clearly that the strong short-range antiferromagnetic $f-f$ correlations are very well described by a long-range ordered state of $f$ electrons, although we know that in $d=1$ this cannot be the exact ground state. Quantitatively similar results may be obtained from a slave-boson mean-field theory with long-range antiferromagnetic correlations. ${ }^{24}$

Even more interesting than the site-independent square of the local magnetic moment of the $f$ electrons $\left\langle\hat{m}_{z}^{2}\right\rangle$ is the actual local magnetic moment of the $c$ and $f$ electrons $\left\langle\hat{m}_{\mathbf{j}, z}^{c, f}\right\rangle$, given by Eqs. (20a) and (20b). They become manifestly site dependent above the transition to the anti-

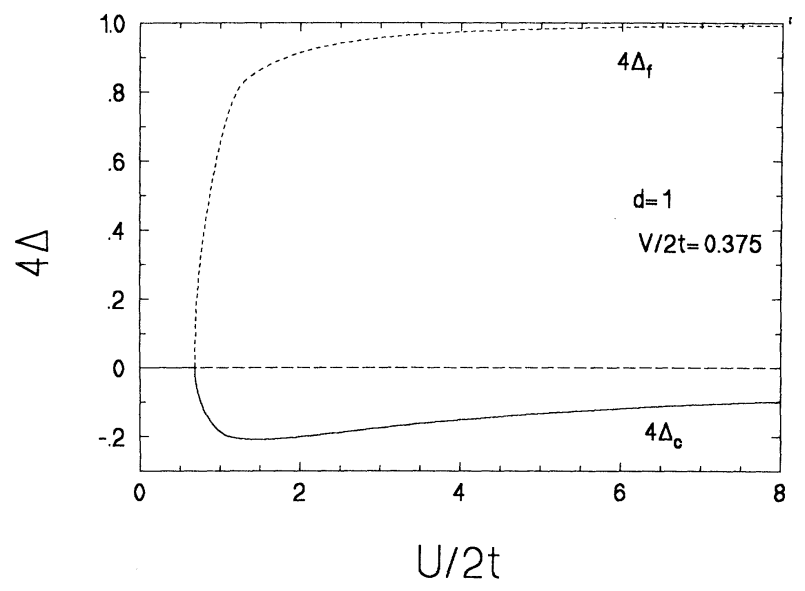

FIG. 4. Amplitudes of the local magnetic moments of $c$ and $f$ electrons, $4 \Delta_{c, f}$ vs $U / 2 t$ in $d=1$ for $V / 2 t=0.375$ (see text). 
ferromagnetically ordered state. In Fig. 4 we show the interaction dependence of the amplitudes of the local magnetic moments $4 \Delta_{c, f}$ of $c$ and $f$ electrons for $d=1$ (the results for $d>1$ are qualitatively similar). Below $U / 2 t \simeq 0.8$, i.e., in the paramagnetic state, the local magnetic moments are identically zero. Above $U / 2 t \simeq 0.8$, $\Delta_{c}$ and $\Delta_{f}$ become nonzero. In particular, $4 \Delta_{f}$ rises rapidly with $U$ and, at $U / 2 t \simeq 2-3$, almost saturates at its maximal values of 1 . Hence for $U / 2 t \gtrsim 2$ the $f$ electrons are ordered in a SDW with an alternating local moment of \pm 1 . By contrast, the amplitude of the $c$-electronSDW, $4 \Delta_{c}$, is considerably smaller (always less than $20 \%$ of $4 \Delta_{f}$ ) and reaches a shallow maximum at the $U$ value where $\Delta_{f}$ starts to saturate. It slowly decreases to zero for increasing $U$, since $U \rightarrow \infty$ effectively corresponds to $V \rightarrow 0$ in which case the $c$ electrons decouple from the $f$ electrons. In particular, the $c$-electron moment is always

(a)

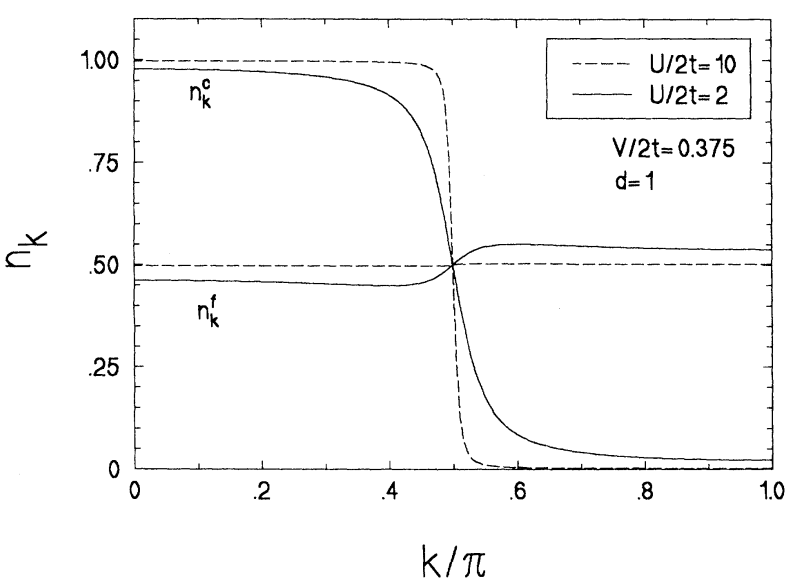

(b)

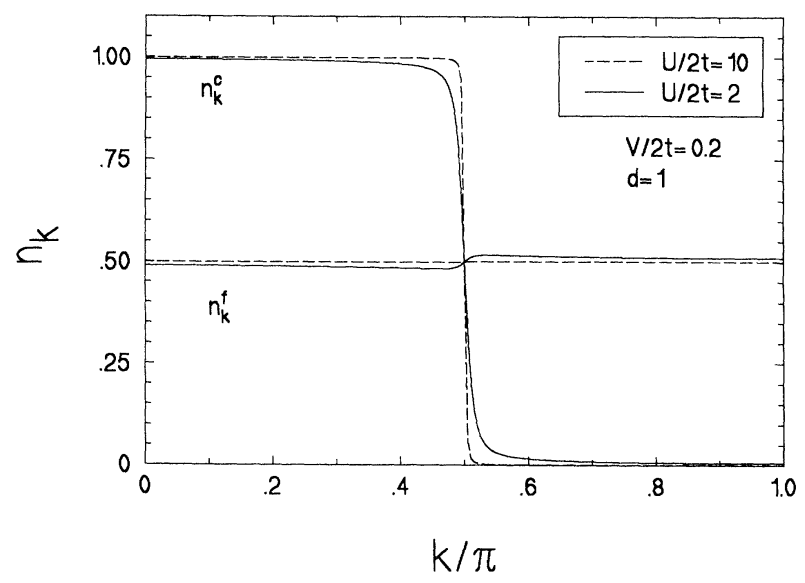

FIG. 5. Momentum distributions $n_{\mathrm{k}}^{c, f}$ of $c$ and $f$ electrons in $d=1$ for two values of $U$ : (a) $V / 2 t=0.375$; (b) $V / 2 t=0.2$. (a)

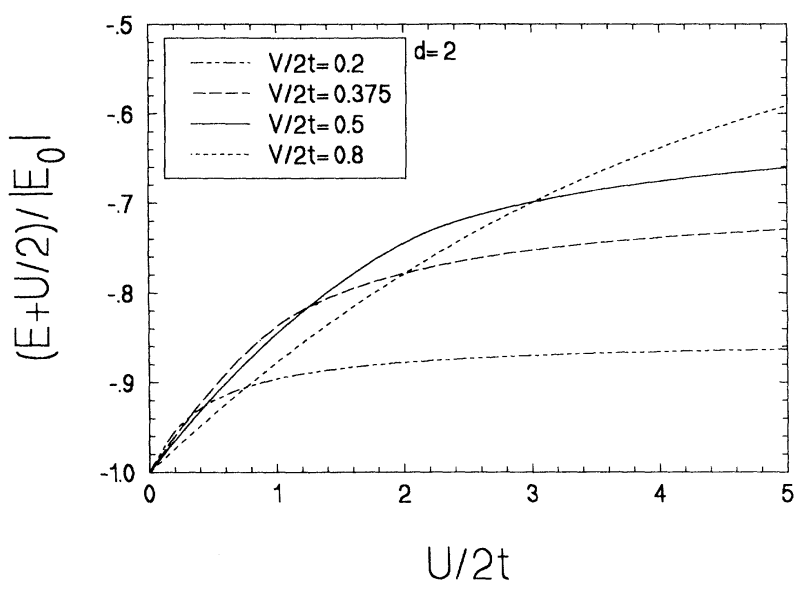

(b)

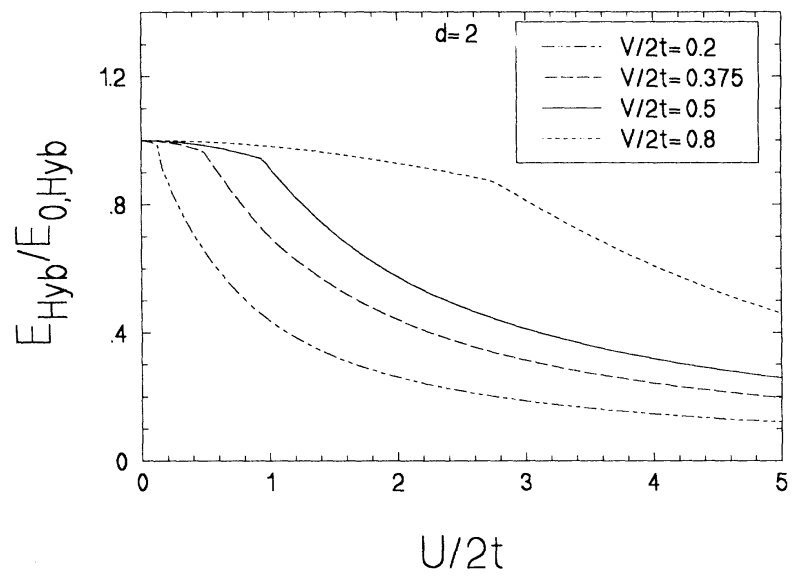

(c)

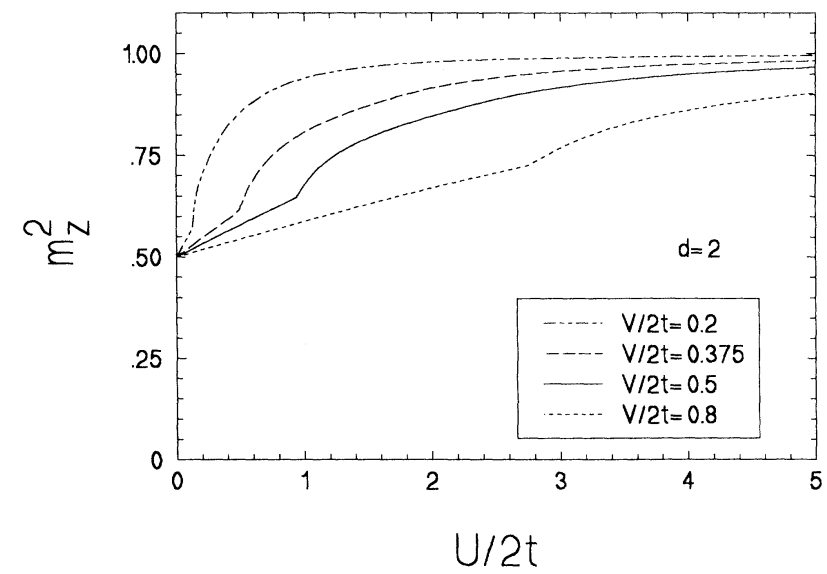

FIG. 6. Same plot as in Fig. 1; evaluation for $d=2$. 
(a)

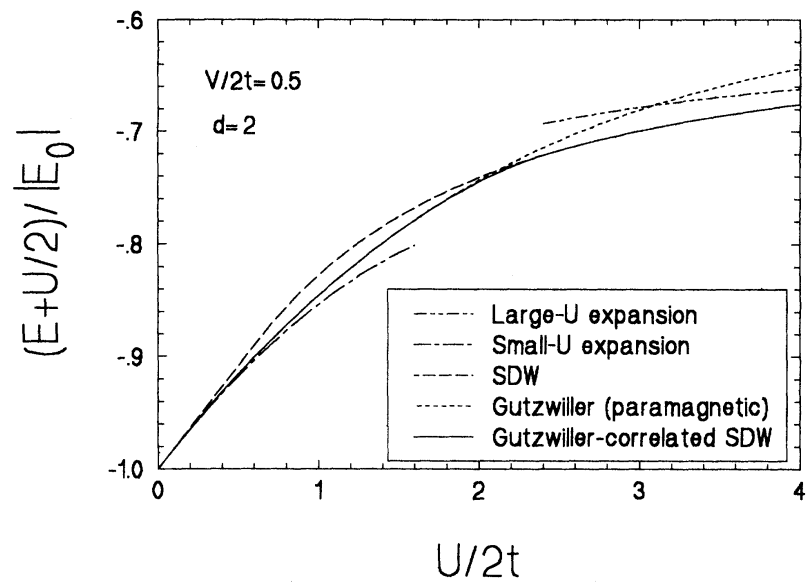

(b)

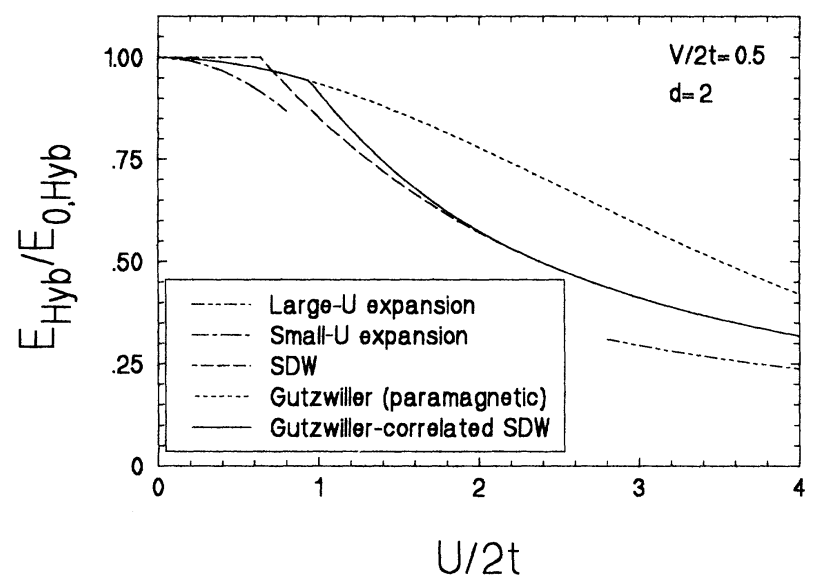

(c)

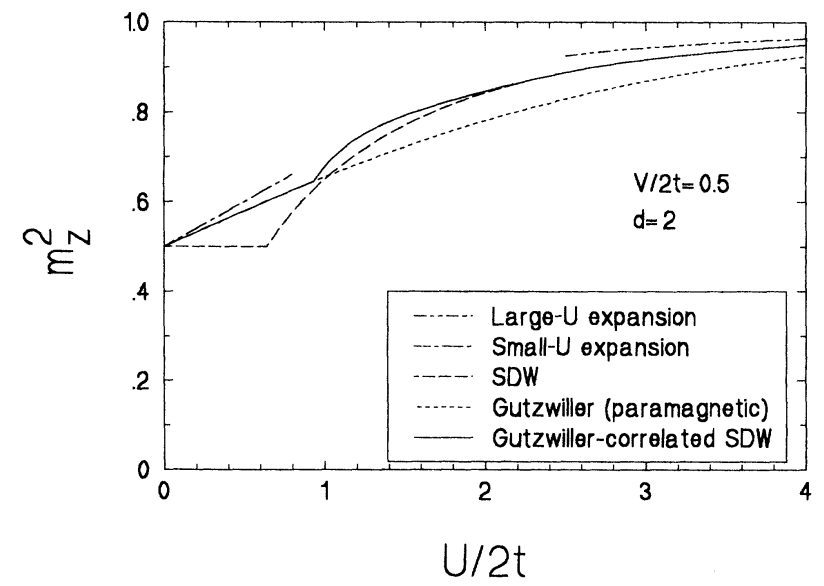

FIG. 7. Same plot as in Fig. 2; evaluation for $d=2$. (a)

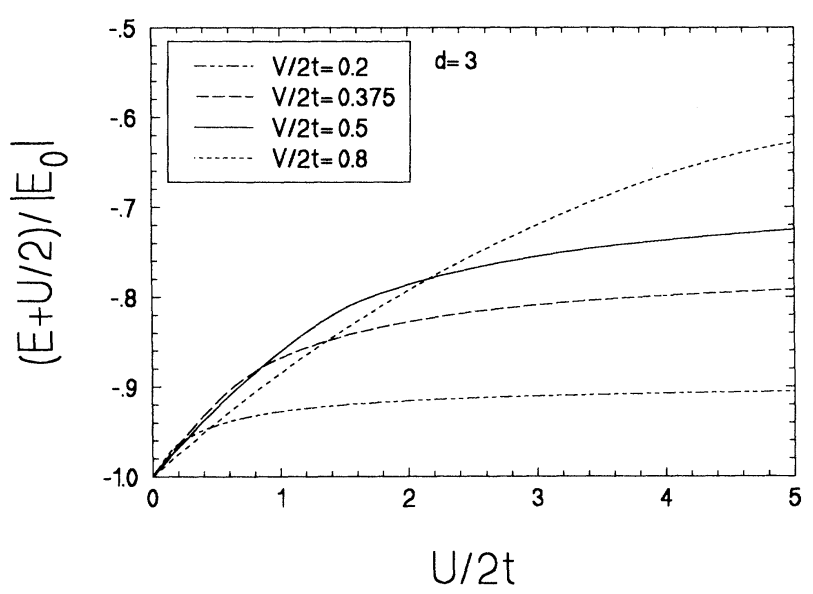

(b)

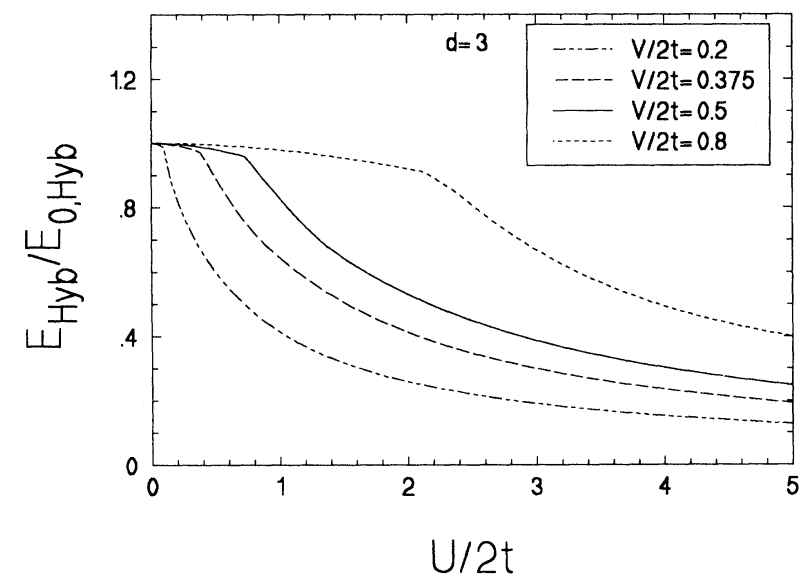

(c)

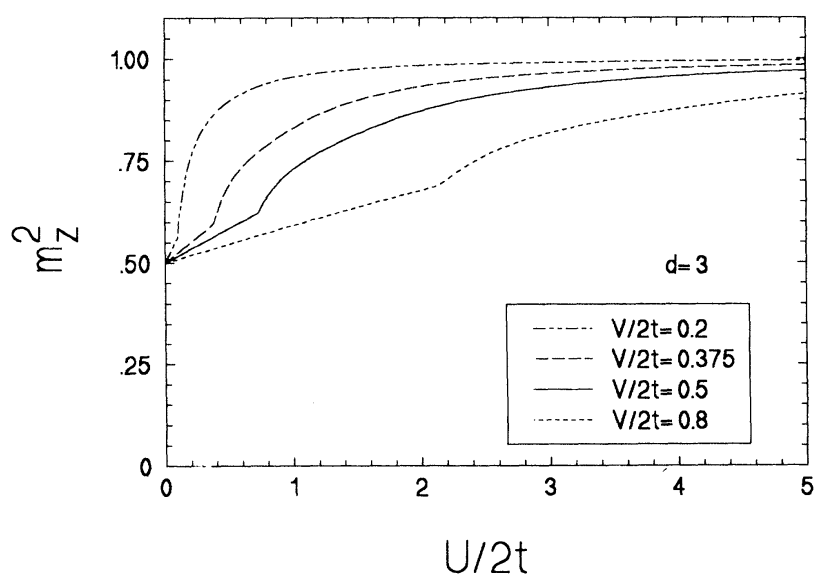

FIG. 8. Same plot as in Fig. 1; evaluation for $d=3$. 
(a)

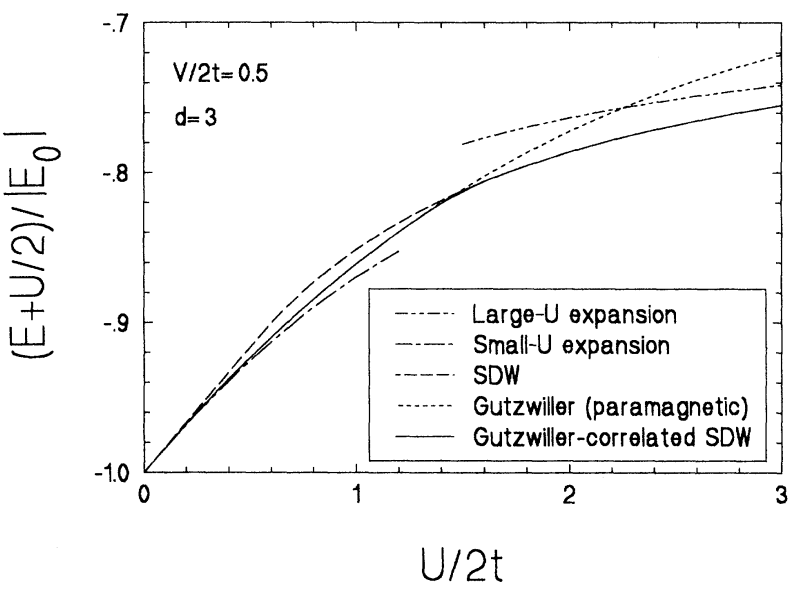

(b)

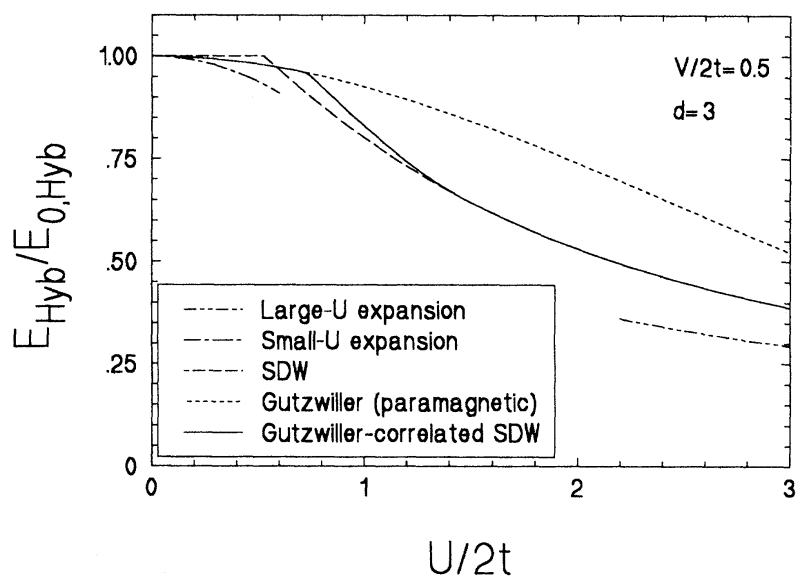

(c)

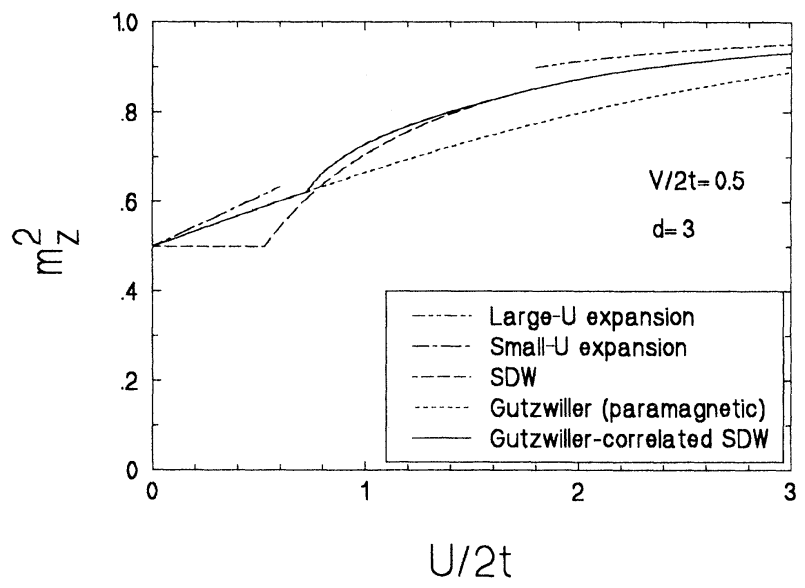

FIG. 9. Same plot as in Fig. 2, evaluation for $d=3$. oriented opposite to the $f$-electron moment. These results are interesting in view of the question concerning the compensation of $f$ spins in the PAM and in its large- $U$ limit, the so-called Kondo lattice model $(\mathrm{KLM}){ }^{25}$ There are two (partly related) issues involved: the compensation on a local and on a global scale. Ueda, Tsunetsugu, and Sigrist ${ }^{26}$ proved rigorously that at half filling the ground state of the symmetric PAM is a total singlet. The result obviously applies also to the KLM at small antiferromagnetic exchange coupling, $J=-8 V^{2} / U$. This compensation is a global one (formation of a "collective singlet"). Furthermore, for large $|J|$, when each $f$ spin is screened anyway, the ground state is a superposition of local singlets and hence is a total singlet, too. Numerical studies clearly show that in the PAM the compensation of the $f$-electron moment is due to intersite correlations, in particular due to antiferromagnetic correlations between the $f$ electrons themselves, ${ }^{12,27}$ and that the correlation between $c$ - and $f$-electron moments is only small and negative. ${ }^{28}$ The importance of magnetic intersite correlations between the $f$ electrons in the PAM and of the collective nature of the compensation effect was also observed in previous variational treatments. ${ }^{29,14}$

Our results are in agreement with those found numerically: (i) the ground state is a total singlet for all $U$ values, the compensation being due to the (long-range) antiferromagnetic order of the $f$ electrons, i.e., a collective effect, (ii) in the ordered phase the moments of the $c$ electrons are always oriented opposite to the $f$ moments but compensate only a fraction ( $\lesssim 20 \%$ ) of the latter.

In Fig. 5 the results for the momentum distributions of the $c$ and $f$ electrons are shown for different $U$ and $V$ values in $d=1$. For the $U$ values chosen here, i.e., for $U / 2 t \gtrsim 2$, the correlation operator in (1) is found to have no more effect. The curves are qualitatively similar to those obtained with the paramagnetic Gutzwiller wave function at $U=\infty .{ }^{29,20}$ Apparently these quantities are not sensitive to the microscopic details of the wave function.

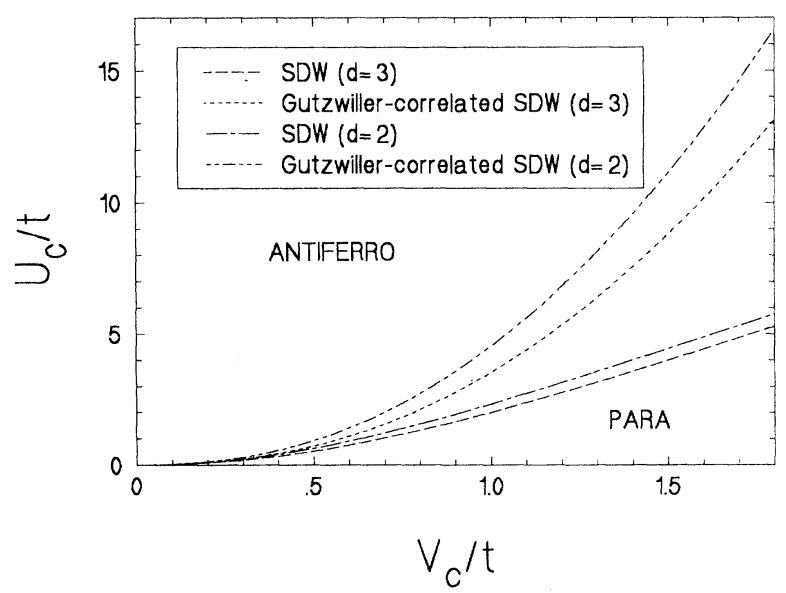

FIG. 10. Magnetic phase diagram for $d=2,3$ as obtained with the uncorrelated and the Gutzwiller-correlated SDW, respectively. 
Having shown that (i) our approach for the evaluation of expectation values in terms of Gutzwiller-correlated wave functions in $d$ dimensions (which becomes exact in the limit $d \rightarrow \infty$ ) gives quantitatively reliable results even in the most unfavorable case, i.e., $d=1$, and that (ii) a variational wave function with long-range order of the $c$ and $f$ electrons gives excellent results even in $d=1$ where true long-range order is not possible at all, we may expect that our results for $d=2$ and 3 will be even more accurate. In Figs. 6 to 9 the quantities $E, E_{\mathrm{Hyb}}$, and $\left\langle\hat{m}_{z}^{2}\right\rangle$ are shown as function of $U$ for various values of the hybridization for $d=2$ and $d=3$, respectively. Qualitatively the same behavior as in $d=1$ is found. In Figs. 7 and 9 these results are compared with perturbation theory ${ }^{12}$ at small and large $U$ as well as with the results obtained with the uncorrelated SDW and the paramagnetic Gutzwiller wave function. So far, numerically exact results are not available for $d=2$ and 3 . Hence our variational results may serve as benchmarks for future numerical work.

In Fig. 10 the magnetic phase diagram of the PAM for $d=2$ and 3 as a function of interaction versus hybridization strength is shown. The lines separate the phase with antiferromagnetic ordering of $f$ spins and $c$ electrons (above the line), from the paramagnetic phase (below the line). A transition is found for all values of the hybridization; however, only in $d>1$ can such a phase transition occur at all. The inclusion of two-particle correlation effects is found to have a considerable effect on the precise position of the phase transition line: for given $V$ the lines are shifted to higher $U$ values. It will be very interesting to compare the above results with quantum Monte-Carlo calculations in $d>1$ which, hopefully, become possible in the near future.

\section{ACKNOWLEDGMENTS}

We gratefully acknowledge useful discussions with Dr. W. Brenig and Professor Dr. E. Müller-Hartmann. One of us (Z.G.) wishes to thank the Alexander von Humbold Foundation for financial support. R.S. gratefully acknowledges financial support from the Studienstiftung des Deutschen Volkes. This work was supported in part by the Sonderforschungsbereich Nr. 341 of the Deutsche Forschungsgemeinschaft.

\section{APPENDIX: MINIMIZATION OF THE ENERGY}

We wish to determine the minimum of the ground-state energy $E$ (24). Differentiating with respect to the $\mathbf{k}$ dependent variational parameters yields the following linear equation

$$
\underline{\underline{M}}_{\mathrm{k}} \underline{x}_{\mathrm{k}}=0 \text {, }
$$

where

$$
\underline{x}_{\mathrm{k}}=\left(v_{\mathrm{k}}, w_{\mathrm{k}}, x_{\mathrm{k}}, u_{\mathrm{k}}, z_{\mathrm{k}}\right),
$$

$$
\underline{\underline{M}}_{\mathbf{k}}=\left[\begin{array}{ccccc}
-\left(\epsilon_{\mathrm{k}}+\lambda_{\mathrm{k}}\right) & \tilde{\lambda}_{\mathrm{k}} & 2 \bar{V} & 0 & -2 \bar{\Delta} \\
\tilde{\lambda}_{\mathrm{k}} & \left(\epsilon_{\mathrm{k}}-\lambda_{\mathrm{k}}\right) & -2 \bar{V} & -2 \bar{\Delta} & 0 \\
2 \bar{V} & -2 \bar{V} & 2\left(\tilde{\lambda}_{\mathrm{k}}-\lambda_{\mathrm{k}}\right) & 0 & 0 \\
0 & -2 \bar{\Delta} & 0 & \left(\epsilon_{\mathrm{k}}-\lambda_{\mathrm{k}}\right) & -\tilde{\lambda}_{\mathrm{k}} \\
-2 \bar{\Delta} & 0 & 0 & -\tilde{\lambda}_{\mathrm{k}} & -\left(\epsilon_{\mathrm{k}}+\lambda_{\mathrm{k}}\right)
\end{array}\right] \text {, }
$$

and

$$
\begin{aligned}
& \bar{V}=V \sqrt{q}, \\
& \bar{\Delta}=V \frac{\partial \sqrt{q}}{\partial \Delta_{f}} \frac{1}{L} \sum_{\mathbf{k}}^{\prime} \frac{x_{\mathbf{k}}\left(w_{\mathbf{k}}-v_{\mathbf{k}}\right)}{N_{\mathbf{k}}} .
\end{aligned}
$$

Nontrivial solutions can be obtained only if $\operatorname{Det}\left(\underline{\underline{M}}_{\mathrm{k}}\right)=0$, leading to the following equation

$$
\left(\tilde{\lambda}_{\mathbf{k}}-\lambda_{\mathbf{k}}\right)\left[\left(\epsilon_{\mathrm{k}}^{2}+\tilde{\lambda}_{\mathrm{k}}^{2}-\lambda_{\mathrm{k}}^{2}\right)\left(\epsilon_{\mathrm{k}}^{2}+4 \bar{V}^{2}+\tilde{\lambda}_{\mathbf{k}}^{2}-\lambda_{\mathrm{k}}^{2}\right)+8 \bar{\Delta}^{2}\left(\tilde{\lambda}_{\mathrm{k}}^{2}-\lambda_{\mathrm{k}}^{2}-\epsilon_{\mathrm{k}}^{2}\right)+16 \bar{\Delta}^{2}\left(\bar{\Delta}^{2}+\bar{V}_{2}\right)\right]=0 .
$$

The k-dependent parameters are given by

$$
\begin{aligned}
& u_{\mathbf{k}}=\frac{8 \bar{\Delta}^{2} \bar{V}^{2}+\tilde{\lambda}_{\mathbf{k}}\left(\tilde{\lambda}_{\mathbf{k}}-\lambda_{\mathbf{k}}\right)\left[\epsilon_{\mathbf{k}}^{2}-\lambda_{\mathbf{k}}^{2}+\tilde{\lambda}_{\mathbf{k}}^{2}+4\left(\bar{V}^{2}+\bar{\Delta}^{2}\right)\right]}{2 \bar{\Delta} \bar{V} \tilde{N}_{\mathbf{k}}} x_{\mathbf{k}}, \\
& v_{\mathbf{k}}=\frac{2 \bar{V}^{2}\left(\epsilon_{\mathbf{k}}-\lambda_{\mathbf{k}}+\tilde{\lambda}_{\mathbf{k}}\right)+\left(\tilde{\lambda}_{\mathbf{k}}-\lambda_{\mathbf{k}}\right)\left[4 \bar{\Delta}^{2}+\tilde{\lambda}_{\mathbf{k}}^{2}-\left(\epsilon_{\mathbf{k}}-\lambda_{\mathbf{k}}\right)^{2}\right]}{\bar{V} \widetilde{N}_{\mathbf{k}}} x_{\mathbf{k}},
\end{aligned}
$$




$$
\begin{aligned}
& z_{\mathbf{k}}=\frac{-8 \bar{\Delta}^{2} \bar{V}^{2}-\left(\tilde{\lambda}_{\mathbf{k}}-\lambda_{\mathbf{k}}\right)\left[4 \bar{\Delta}^{2}\left(\epsilon_{\mathbf{k}}+\lambda_{\mathbf{k}}\right)-\left(\epsilon_{\mathbf{k}}-\lambda_{\mathbf{k}}\right)\left(\epsilon_{\mathbf{k}}^{2}-\lambda_{\mathbf{k}}^{2}+\tilde{\lambda}_{\mathbf{k}}^{2}+4 \bar{V}^{2}\right)\right]}{2 \bar{\Delta} \bar{V} \tilde{N}_{\mathbf{k}}} x_{\mathbf{k}}, \\
& w_{\mathbf{k}}=\frac{2 \bar{V}^{2}\left(\epsilon_{\mathbf{k}}-\lambda_{\mathbf{k}}+\tilde{\lambda}_{\mathbf{k}}\right)+2 \tilde{\lambda}_{\mathbf{k}}\left(\tilde{\lambda}_{\mathbf{k}}-\lambda_{\mathbf{k}}\right) \epsilon_{\mathbf{k}}}{\bar{V}_{\mathbf{N}}} x_{\mathbf{k}},
\end{aligned}
$$

where

$$
\widetilde{N}_{\mathbf{k}}=\epsilon_{\mathbf{k}}^{2}+\lambda_{\mathbf{k}}^{2}-\tilde{\lambda}_{\mathbf{k}}^{2}-4 \bar{\Delta}^{2}-2 \epsilon_{\mathbf{k}}\left(\lambda_{\mathbf{k}}-\tilde{\lambda}_{\mathbf{k}}\right)
$$

The minimization with respect to $d^{f}$ yields one more equation

$$
U=8 \bar{\Delta} \frac{\partial \sqrt{q}}{\partial d^{f}} / \frac{\partial \sqrt{q}}{\partial \Delta_{f}} .
$$

From Eq. (23b) and Eq. (A4) the Lagrange multipliers are found as

$$
\begin{aligned}
& \tilde{\lambda}_{\mathbf{k}}=0 \\
& \lambda_{\mathbf{k}}=-\left(2 \bar{V}^{2}+4 \bar{\Delta}^{2}+\epsilon_{\mathrm{k}}^{2}+2 \sqrt{\bar{V}^{4}+4 \bar{\Delta}^{2} \epsilon_{\mathrm{k}}^{2}}\right)^{1 / 2} .
\end{aligned}
$$

This corresponds to the minimum of the energy. The minimal energy is given by (25).

${ }^{1}$ H. R. Ott, in Progress in Low Temperature Physics, edited by D. F. Brewer (North-Holland, Amsterdam, 1987), Vol. XI, p. 215.

${ }^{2}$ Note that, in general, the hybridization matrix element should be a k-dependent quantity because an on-site hybridization is forbidden for parity reasons. Hence $V_{\mathbf{k}}$ should have a different symmetry than $\epsilon_{\mathrm{k}}$ [G. Czycholl, Phys. Rep. 143, 277 (1986)].

${ }^{3}$ It was recently shown by one of us [R. Strack, Phys. Rev. Lett. 70, 833 (1993)] that for $U=\infty, n=2$ the asymmetric PAM can be solved exactly in $d=1$, if the $f$ electrons have a dispersion, too, and the parameters are restricted by $t^{\prime}=V^{2} / t$, $E_{f}=2 t-4 V^{2} / t$. Here $t$ and $t^{\prime}$ are the hopping matrix elements of the $c$ and $f$ electrons, respectively. The exact ground-state energy $E$ is then simply given by $E / L=-4 V^{2} / t$, where $L$ is the number of lattice sites.

${ }^{4}$ M. C. Gutzwiller, Phys. Rev. Lett. 10, 159 (1963); Phys. Rev. A 137, 1726 (1965).

${ }^{5}$ T. M. Rice and K. Ueda, Phys. Rev. Lett. 55, 995 (1985); 55, 2093(E) (1985); Phys. Rev. B 34, 6420 (1986).

${ }^{6}$ B. H. Brandow, Phys. Rev. B 33, 215 (1986).

${ }^{7}$ P. Fazekas, J. Magn. Magn. Mater. 63\&64, 545 (1987).

${ }^{8}$ D. Vollhardt, Rev. Mod. Phys. 56, 99 (1984).

${ }^{9}$ W. Metzner and D. Vollhardt, Phys. Rev. Lett. 62, 324 (1989).

${ }^{10} \mathrm{~F}$. Gebhard and D. Vollhardt, in Interacting Electrons in Reduced Dimensions, edited by D. Baeriswyl and D. Campbell (Plenum, New York, 1989), p. 123.

${ }^{11} \mathrm{~K}$. Yosida and A. Yoshimori, in Magnetism, edited by H. Suhl (Academic, New York, 1973), Vol. 5, p. 253.

${ }^{12}$ R. Blankenbecler, J. R. Fulco, W. Gill, and D. J. Scalapino, Phys. Rev. Lett. 58, 411 (1987).

${ }^{13}$ R. Strack, Diploma thesis, RWTH Aachen, 1990 (unpublished).
${ }^{14}$ H. Shiba and P. Fazekas, Prog. Theor. Phys. Suppl. 101, 403 (1990).

${ }^{15}$ R. Strack and D. Vollhardt, Mod. Phys. Lett. B 2, 1377 (1991).

${ }^{16}$ W. Brenig and E. Müller-Hartmann, Ann. Phys. 1, 39 (1992).

${ }^{17}$ W. Metzner and D. Vollhardt, Phys. Rev. Lett. 62, 324 (1989).

${ }^{18}$ W. Metzner, Z. Phys. B 77, 253 (1989).

${ }^{19}$ F. Gebhard, Phys. Rev. B 41, 9452 (1990).

${ }^{20}$ R. Strack and D. Vollhardt, J. Low Temp. Phys. 84, 357 (1991).

${ }^{21}$ W. Metzner and D. Vollhardt, Phys. Rev. Lett. 59, 121 (1987); Phys. Rev. B 37, 7382 (1988).

${ }^{22}$ F. Gebhard, Dissertation thesis, RWTH Aachen, 1990 (unpublished).

${ }^{23}$ W. Metzner and D. Vollhardt, Phys. Rev. B 39, 4462 (1989).

${ }^{24} \mathrm{~B}$. Möller (private communication); this agreement is not surprising, since on the mean-field level the results of the Kotliar-Ruckenstein slave boson formulation are equivalent to those obtained for arbitrary Gutzwiller-type wave functions in $d=\infty$ (Refs. 17-19). Hence, in the absence of longrange order the results are necessarily identical to the ones obtained here, since both approaches start from the (paramagnetic) Fermi sea of electrons in this case. On the other hand, in the presence of long-range order the agreement will depend on the details of the assumed order in the two approaches.

25P. Nozières, Ann. Phys. (Paris) 10, 19 (1985).

${ }^{26}$ K. Ueda, H. Tsunetsugu, and M. Sigrist, Phys. Rev. Lett. 68, 1030 (1992).

${ }^{27}$ T. A. Costi, E. Müller-Hartmann, and K. A. Ulrich, Solid State Commun. 66, 343 (1988).

${ }^{28}$ K. Ueda, J. Phys. Soc. Jpn. 58, 3465 (1989).

${ }^{29}$ H. Shiba, J. Phys. Soc. Jpn. 55, 2765 (1986). 